CNS Spectrums (2019), 24, 609-615. (C) Cambridge University Press 2019. This is an Open Access article, distributed under the terms of the Creative Commons Attribution licence (http://creativecommons.org/licenses/by/4.0/), which permits unrestricted re-use, distribution, and reproduction in any medium, provided the original work is properly cited.

doi:10.1017/S1092852918001645

\title{
Abnormalities of striatal morphology in gambling disorder and at-risk gambling
}

\author{
Jon E. Grant, ${ }^{1 *}$ (1) Masanori Isobe, ${ }^{2,3}$ and Samuel R. Chamberlain ${ }^{2}$ (]
}

\author{
${ }^{1}$ Department of Psychiatry \& Behavioral Neuroscience, University of Chicago, Chicago, IL, USA \\ ${ }^{2}$ Department of Psychiatry, University of Cambridge; \& Cambridge and Peterborough NHS Foundation Trust (CPFT), UK \\ ${ }^{3}$ Department of Neuropsychiatry, Faculty of Medicine, University of Tokyo Hospital, \& Nippon Foundation International Fellowship, Japan
}

Objective. The clinical phenotype of gambling disorder (GD) is suggestive of changes in brain regions involved in reward and impulse suppression, notably the striatum. Studies have yet to characterize striatal morphology (shape) in GD and whether this may be a vulnerability marker.

Aims. To characterize the morphology of the striatum in those with disordered gambling (at-risk gambling and GD) versus controls.

Method. Individuals aged 18-29 years were classified a priori into those with some degree of GD symptoms (at-risk gambling and GD) or controls. Exclusion criteria were a current mental disorder (apart from GD), history of brain injury, or taking psychoactive medication within 6 weeks of enrollment. History of any substance use disorder was exclusionary. Participants completed an impulsivity questionnaire and structural brain scan. Group differences in volumes and morphology were characterized in subcortical regions of interest, focusing on the striatum.

Results. Thirty-two people with GD symptoms (14 at-risk and 18 GD participants) and 22 controls completed the study. GD symptoms were significantly associated with higher impulsivity and morphological alterations in the bilateral pallidum and left putamen. Localized contraction in the right pallidum strongly correlated with trait impulsivity in those with GD symptoms.

Conclusions. Morphologic abnormalities of the striatum appear to exist early in the disease trajectory from subsyndromal gambling to GD and thus constitute candidate biological vulnerability markers, which may reflect differences in brain development associated with trait impulsivity. Striatal morphology and associated impulsivity might predispose to a range of problematic repetitive behaviors.

Received 2 October 2018; Accepted 22 November 2018; First published 18 March 2019

Key words: gambling, morphology, neuroimaging.

\section{Introduction}

Gambling disorder is a significant public health problem affecting $0.4 \%$ to $1 \%$ of the US population and is also prevalent in many other countries. ${ }^{1}$ Understanding the chain of progression from recreational gambling to gambling disorder is vital toward understanding the underlying biological mechanisms (pathogenesis). Comparing

*Address correspondence to: Jon E. Grant, Department of Psychiatry \& Behavioral Neuroscience, University of Chicago, Pritzker School of Medicine, 5841 S. Maryland Avenue, MC 3077, Chicago, IL, USA. (Email: jongrant@uchicago.edu)

This research was supported by a Center of Excellence in Gambling Research grant from the National Center for Responsible Gaming and a Wellcome Trust Clinical Fellowship (110049/Z/15/Z). people with gambling disorder with those at an increased risk of developing gambling disorder would help to elucidate whether neurobiological aspects of gambling disorder are evident prior to the development of overt pathology or stem from the disorder itself, perhaps even reflecting the harmful effects of recurrent gambling on brain function.

Gambling disorder can be conceptualized from a neurobiological perspective in terms of an excessive drive from the subcortical regions involved in reward processing, coupled with diminished top-down control from the prefrontal cortical regions. ${ }^{2,3}$ Consistent with this perspective, people with gambling disorder often exhibit impairments across a spread of cognitive domains including inhibitory 
control, working memory, and decision-making. ${ }^{3-6}$ Extensive translational data implicate subcortical structures in these abilities. ${ }^{7}$

Functional neuroimaging (fMRI) studies involving healthy volunteers have demonstrated that the striatum and pallidum respond to reward, in particular, through dopaminergic signaling, and encode reward expectancy (anticipation of reward). ${ }^{8}$ Such subcortical structures are also central to contemporary computational models of decision-making, which, in turn, stem from human (and translational) data. ${ }^{9,10}$ Gambling disorder is conceptualized as a Substance-Related and Addictive Disorder in DSM-5, and abnormalities of decision-making are central to understanding its symptomatology (eg, loss of control, craving, escalation of reward-seeking over time, and neglect of other areas of life). Reward-related increases in striatal dopamine release have been found in gambling disorder, ${ }^{11}$ along with a positive correlation between such dopamine release and symptom severity. ${ }^{12}$ Functional imaging has also indicated that gambling disorder is typically associated with blunted mesolimbic-prefrontal cortex responses to general rewards but heightened activation to gambling-related stimuli. ${ }^{13}$ An fMRI study of gambling disordered adults $(n=10)$ using a monetary reward task demonstrated that gamblers, as compared with controls, exhibited decreased neural activity in the pallidum for decision-making under risk, as opposed to decision under ambiguity, and increased neural activity within the putamen prior to bet choices, as opposed to safe choices. ${ }^{14} \mathrm{~A}$ subset of patients with Parkinson's disease develops impulsive symptoms, including gambling disorder, due to pro-dopaminergic therapy. ${ }^{15}$ In functional imaging, such impulsive symptoms in Parkinson's disease were associated with heightened connectivity from the ventral striatum to the putamen and pallidum. ${ }^{16}$

Though striatal brain regions are demonstrably involved in decision-making ${ }^{8}$ and functional imaging data have identified altered responses to reward in such regions in gambling disorder, ${ }^{13}$ direct quantification of structural changes in these regions in patients is lacking. One voxel-based morphology (VBM) investigation found that gambling disorder was associated with increased gray matter volumes in the ventral striatum. ${ }^{17,18}$ Another VBM study examining 30 male never-treated gambling patients and 30 controls showed increased absolute global gray matter volumes in gamblers relative to controls, as well as relatively decreased volumes in the left putamen. ${ }^{19}$

Subcortical structures are difficult to visualize structurally accurately with conventional pipelines because of poor, heterogeneous signal intensities. ${ }^{20}$ Typical imaging analysis pipelines were designed for analysis of the cortex rather than the subcortical regions. ${ }^{21,22}$ Neuroimaging pipelines are now available that enable the sensitive measurement of localized differences in deformations of subcortical structure shapes across groups. This latter approach of examining localized abnormalities in subcortical brain structure (ie, quantification of local curvature) has the advantage of not relying on arbitrary smoothing extent or tissue classification. ${ }^{20}$ This innovative modeling approach has been shown to be sensitive to pathologies in other contexts, such as in Alzheimer's Disease. ${ }^{20}$

Therefore, the current study aimed to examine whether localized morphometric differences of the striatum (caudate, putamen, accumbens, and pallidum) exist in people with gambling disorder symptoms, compared with recreational non-pathologic gambler controls (ie, those with no gambling disorder symptoms). We hypothesized, based on the abovementioned literature, that these regions would show abnormal morphology in those with symptoms, which, in turn, would correlate with the extent of symptoms and impulsivity more broadly.

\section{Method}

\section{Subjects}

Participants were recruited using media advertisements for anyone who had gambled within the past year. Inclusion criteria were: age 18-29 years (this was set to limit the confounding effects of age), right-handedness, no use of psychotropic medications in the past 6 weeks, and no contraindication to MRI. Participants with current mental disorders (apart from gambling disorder in the gambling disorder group) including any other impulse control disorder or a lifetime history of psychotic disorder, bipolar disorder, or substance use disorder were excluded. Healthy controls were recruited using media advertisements based on no lifetime or current psychiatric disorders.

The study procedures were carried out in accordance with the ethical standards laid out in the Declaration of Helsinki. The University of Chicago Institutional Review Board approved the study and consent procedures. After providing a complete description of the study to the subjects, written informed consent was obtained.

\section{Assessments}

Demographic variables including age and gender were recorded for all participants. Subjects underwent a psychiatric evaluation, which included the Structured Clinical Interview for Pathological Gambling (SCI-PG) ${ }^{23}$ adapted for DSM-5. ${ }^{24}$ A score of 0 on the SCI-PG designated controls, a score of 1-3 defined a participant as being an at-risk gambler, and a score of 4 or greater was consistent with meeting criteria for gambling disorder.

Clinical measures included: Mini International Neuropsychiatric Inventory (MINI), ${ }^{25}$ the Eysenck 
Impulsivity Questionnaire (EIQ), ${ }^{26}$ and the National Adult Reading Test. ${ }^{27}$

After completing these assessments, participants undertook high-resolution structural imaging using a 3-Tesla (3T) scanner with magnetization-prepared rapid gradient echo (3D-MPRAGE) sequences. Axial 3-dimensional T1-weighted scans were acquired using the following parameters: repetition time $=2000 \mathrm{~ms}$, echo time $=3.0 \mathrm{~ms}$, flip angle $=9$ degrees, field of view $=256 \times$ 256 , and resolution $=1 \times 1 \times 1 \mathrm{~mm}$ ).

\section{Statistical analysis, including neuroimaging processing steps}

Group differences in demographic and clinical measures were explored using independent sample $t$ tests and chi-square tests $(\mathrm{P}<0.05$, uncorrected $)$ in SPSS v24.0.

Image preprocessing and data extraction were undertaken using the University of Chicago Midway computing system. We employed the same methodology as with a previous publication by our group. ${ }^{28} \mathrm{~T} 1$-weighted images were automatically bias-field corrected and nonlinearly registered to the MNI 152 standard space. We employed the Integrated Registration and Segmentation Tool (FIRST) of FMRIB implemented in FSL 5.0.9 to automatically segment subcortical structures. ${ }^{20}$ Segmentation was based on shape models with structural boundaries obtained from 336 manually segmented images and resulted in a deformable surface mesh of each subcortical structure consisting of vertices. The meshes were reconstructed and filled in the MNI space, and boundary correction was applied. Then, the segmented images were transformed into the original space. All segmented images were visually checked for errors in registration and segmentation.

A region of interest approach was used for the neuroimaging analyses. Based on literature pertaining to models of decision-making, findings in gambling disorder, and findings in impulse control disorders in Parkinson's disease (outlined in the introduction), we specifically focused on the striatum, defined as the putamen, caudate, accumbens, and pallidum. We calculated the total intracranial volume (ICV) as the sum of the volumes of gray matter, white matter, and cerebrospinal fluid (CSF) using the Automated Segmentation Tool (FAST) of FMRIB. ${ }^{29}$ Each subject's brain scan was skull-stripped using the Brain Extraction Tool and linearly aligned to the MNI152 space, and the inverse of the determinant of the affine transformation matrix computed by the software was multiplied by the ICV size of the template. We adjusted the subcortical volumes by the intracranial volumes (ICV) of each individual. ${ }^{30}$

Subcortical volumes were compared between those with gambling disorder symptoms (GD $+\mathrm{AR})$ and controls (HC) using $t$ tests. Values were reported uncorrected for multiple comparisons but were only deemed statistically significant if they withstood a Bonferroni correction for the number of comparisons undertaken.

For the morphometric analysis, a vertex analysis implemented in FIRST (FSL) was employed to compare the shapes of the subcortical structures. ${ }^{20} \mathrm{~A}$ negative value of the vertex represented deformation in an inward direction, and a positive value of a vertex indicated deformation in an outward direction. Curvature abnormalities were identified between those with gambling disorder symptoms (GD + AR) and controls (HC) using "Randomise," a permutation-based non-parametric testing method implemented in FSL with 5000 iterations that corrects for multiple comparisons and uses Threshold-Free Cluster Enhancement (TFCE) as recommended. ${ }^{31-33}$ We used a 2-group comparison because the primary interest was in determining abnormalities in those with gambling disorder symptoms collectively compared with controls. If significant regions of morphometric abnormalities were found, correlations were explored with the clinical measures (Eysenck scores and SCIGD scores) using Spearman's rho, with significance set at a $\mathrm{P}$ of $<0.05$. Correlations were reported uncorrected but were only deemed significant if they withstood a Bonferroni correction for the number of correlation analyses undertaken.

\section{Results}

Of the 54 participants, 18 had a gambling disorder, 14 met criteria for being at-risk gamblers, and 22 were controls. None of the participants had ever sought treatment for gambling behavior. Although the participants had no current mental health issues other than gambling disorder, 2 were former smokers (only occasionally), and 3 reported histories of anxiety problems; however, none met the criteria for a previous anxiety disorder.

The demographic and clinical features of the participants with GD, AR, and $\mathrm{HC}$ are presented in Table 1, in which it can be seen that there were no differences in age, gender, or IQ. Regarding gambling behavior, the majority of both gambling groups reported casino gambling as their primary form of gambling, with slots and blackjack as their preferred games (15 [83.3\%] of the gambling disordered participants and 12 [85.7\%] of the at-risk). Gambling disorder symptoms were associated with significantly higher impulsiveness on the Eysenck Impulsiveness measure, as compared with the controls.

\section{Group differences in subcortical volumes and morphology}

Table 2 provides group volumetric differences. There were no significant group differences in volumes in the regions of interest. 
TABLE 1. Demographic and clinical comparison of3 levels of gambling behavior

\begin{tabular}{lcccrr} 
(SD) & HC & AR & GD & Group-wide statistic & P value \\
\hline Number [female] & $22[14]$ & $14[7]$ & $18[6]$ & 4.316 & 0.116 \\
Age & $30.2(12.0)$ & $26.5(2.0)$ & $25.9(2.7)$ & 1.711 & 0.191 \\
NART & $90.4(16.1)$ & $89.1(8.7)$ & $83.4(8.9)$ & 1.725 & 0.193 \\
SCI-PG & $0(0)$ & $1.79(0.80)$ & $6.00(1.94)$ & 128.657 & $<0.001$ \\
EIQ & & & & 7.150 \\
Impulsivity & $4.67(3.60)^{\mathrm{a}, \mathrm{b}}$ & $8.92(4.23)^{\mathrm{b}}$ & $10.22(4.10)^{\mathrm{a}}$ & 2.138 & 0.002 \\
Venturesomeness & $8.33(3.14)$ & $10.21(3.89)$ & $10.67(3.89)$ & 2.836 & 0.131 \\
Empathy & $12.58(2.78)$ & $11.71(3.67)$ & $14.11(2.19)$ & 0.070 \\
\hline
\end{tabular}

AR, at-risk gamblers; EIQ, Eysenck Impulsivity Questionnaire; GD, gambling disorder; HC, healthy controls; NART, National Adult Reading Test; SCI-PG, Structured Clinical Interview for Pathological Gambling; SD, standard deviation.

aP $<0.05$ post hoc analysis between $\mathrm{HC}$ and GD.

${ }^{\mathrm{b} P}<0.05$ post hoc analysis between $\mathrm{HC}$ and $\mathrm{AR}$.

\section{TABLE 2. Volumetric analysis}

\begin{tabular}{lcccc} 
(SD) & $\mathrm{HC}$ & $\mathrm{GD}+\mathrm{AR}$ & $\mathrm{t}$ value & Uncorrected P value \\
\hline GMV & $582670(59003)$ & $580662(61470)$ & 0.120 & 0.905 \\
WMV & $507871(59601)$ & $506506(72851)$ & 0.073 & 0.942 \\
ICV & $1395966(131429)$ & $1396334(165300)$ & 0.009 & 0.993 \\
Lt NAcc & $0.400(0.088)$ & $0.460(0.120)$ & 1.981 & 0.053 \\
Lt caudate & $2.613(0.325)$ & $2.639(0.350)$ & 0.275 & 0.784 \\
Lt pallidum & $1.296(0.135)$ & $1.374(0.256)$ & 1.307 & 0.197 \\
Lt putamen & $3.949(0.553)$ & $4.164(0.485)$ & 1.509 & 0.137 \\
Rt NAcc & $0.317(0.080)$ & $0.305(0.099)$ & 0.474 & 0.637 \\
Rt caudate & $2.639(0.336)$ & $2.686(0.331)$ & 0.505 & 0.616 \\
Rt pallidum & $1.341(0.118)$ & $1.449(0.163)$ & 2.649 & 0.011 \\
Rt putamen & $3.818(0.521)$ & $3.993(0.431)$ & 1.345 & 0.185 \\
\hline
\end{tabular}

AR, at-risk gamblers; GD, gambling disorder; GMV, gray matter volume; HC, healthy controls; ICV, intracranial volume; Lt, left; NAcc, nucleus accumbens; Rt, right; WMV, white matter volume.

There were no significant group differences with the Bonferroni correction.

Values in brackets are standard deviations.

Several significant morphometric abnormalities were associated with a subsyndromal and clinical gambling disorder, as compared with controls. Specifically, localized morphometric abnormalities were found in the bilateral pallidum and left putamen (Figure 1; FDR $\mathrm{P}<0.05$ ). In post hoc $t$ tests using extracted cluster means, at-risk gamblers did not differ significantly from participants with gambling disorder regarding curvature in the identified abnormal significant clusters (all P $>0.10$ ).

\section{The relationship between morphology and clinical measures}

In terms of clinical correlates, the morphological shape of the right pallidum demonstrated a significant correlation with the impulsivity subscale score of the EIQ across all participants pooled; this was because of a significant correlation in those with gambling disorder symptoms, but not in the healthy controls (see Figure 2).
No significant correlation was found in the severity of gambling symptoms.

\section{Discussion}

This study investigated subcortical morphology in individuals with gambling disorder symptoms, as compared with controls who gamble but had no pathologic symptoms. This approach stemmed from our goal to evaluate morphological abnormalities in the subcortical structures common to those with any degree of gambling disorder symptoms versus those with none, as such differences may inform the understanding of vulnerability markers and differences in brain development predisposing persons to these and other impulsive psychiatric problems. Partly consistent with our a priori hypothesis, we identified morphologic abnormalities in the pallidum and putamen in those with gambling disorder symptoms. The morphological abnormalities in the right pallidum 

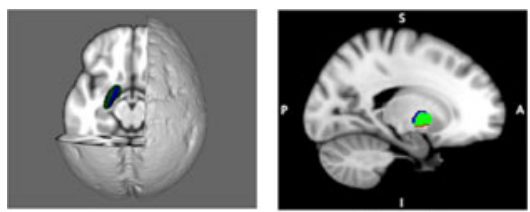

$\mathrm{X}=-18$
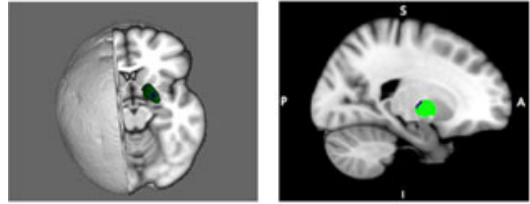

$X=21$
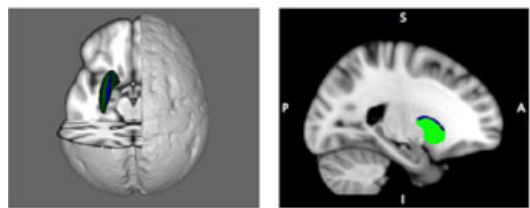

$X=-22$

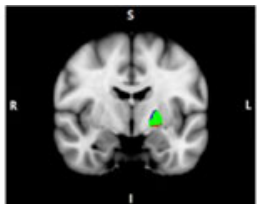

$Y=-6$

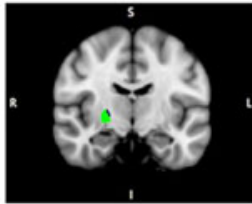

$Y=-11$

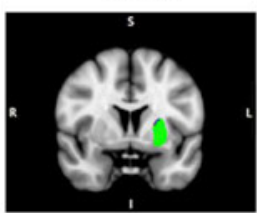

$Y=3$

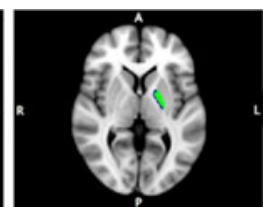

$Z=3$

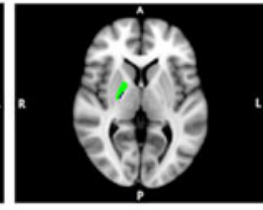

$Z=4$

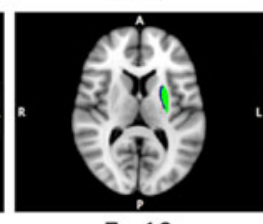

$\mathrm{Z}=10$

FIGURE 1. Morphological abnormalities in disordered gamblers, as compared with controls. Blue indicates a significant excess in the inward curvature, and red indicates a significant excess in the outward curvature in the gambling disorder symptom group versus controls (permuted $\mathrm{P}<0.05$, corrected). Top row, left pallidum; middle row, right pallidum; and bottom row, left putamen.

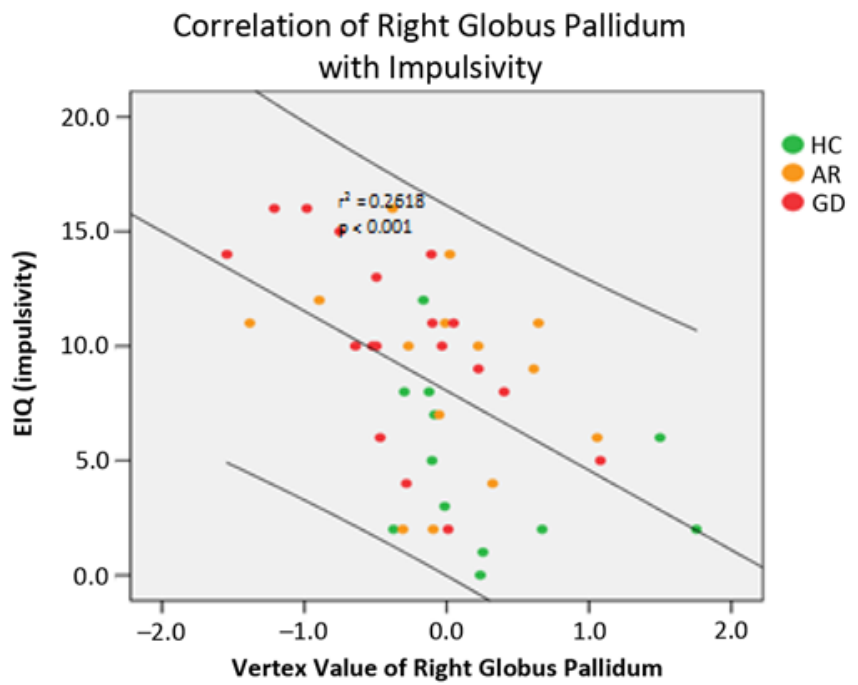

FIGURE 2. Correlation between the mean curvature (vertex value) of the right pallidum in a cluster for group difference and impulsivity on the Eysenck Impulsivity Questionnaire (EIQ). The correlation was significant with all subjects pooled and in the combined GD $+A R$ group $(r=0.45, P<0.001)$, but not in the controls $(r=0.03, P>0.3)$.

correlated highly significantly with impulsivity scores on the EIQ across all study participants. Contrary to expectation, morphology in these structures did not correlate significantly with symptom severity in those with gambling disorder symptoms. We conclude that abnormalities in the striatal morphology, specifically involving the pallidum, are associated with trait impulsivity and thus may predispose persons to gambling disorder and potentially other impulsive symptomatologies.
The striatum encodes reward through dopaminergic signaling, ${ }^{8}$ and aberrant functional connectivity between such structures has been found in impulse control problems in Parkinson's disease, perhaps arising from prodopaminergic therapy. ${ }^{16}$ The current data not only enhance existing neurobiological models of gambling disorder ${ }^{13}$ but also extend beyond these models by suggesting that pallidum abnormalities, in particular, may be a vulnerability marker associated more broadly 
with impulsive tendencies (measured here using the Eysenck questionnaire), rather than necessarily only to disordered gambling per se. Neural responses in the pallidum have been shown to be specific to high reward levels occurring in the context of increasing reward. ${ }^{10}$ Thus, the pallidum may play a key role in the pathophysiology of gambling disorder, and this may offer some explanation for what we see clinically in problem gamblers, that is, the inability to control the behavior despite the negative consequences. ${ }^{34,35}$ Our results suggest that pallidum curvature abnormalities are more related to the extent of trait impulsivity rather than gambling disorder symptoms in particular. In preclinical work, a subset of pallidum neurons has been found to respond selectively in situations requiring the sudden cancellation of impulsive actions. ${ }^{36}$ Therefore, we suggest that morphological abnormalities (versus controls) in the pallidum might contribute to (or correlate with) impulsive traits, which, in turn, may predispose toward a range of problematic behaviors including disordered gambling.

These findings are interesting if also placed in the context of imaging results in the field of substance addiction that are pertinent given that gambling disorder is now regarded as a Substance-Related and Addictive Disorder in DSM-5. For example, bilateral gross symmetric lesions in the pallidum have been associated with heroin intoxication, ${ }^{37}$ and lesions in the pallidum have been found in $5-10 \%$ of heroin-addicted individuals postmortem. ${ }^{38}$ More recently, widespread gray matter reductions in the pallidum (in addition to other areas) have been found in adults undergoing methadone maintenance treatment for opioid use disorder. $^{39}$

This study had several positive features, notably that it is the first imaging study of the subcortical structures in adults with varying levels of gambling severity, and entailed examination of not only the volumes but also the shapes (localized curvature) of the implicated structures. Of course, we examined only localized morphometric differences in the striatum, and, therefore, whole brain analyses may have resulted in additional findings. We focused on the striatum for several reasons including a relative lack of data on this region in gambling disorder; its likely involvement in reward and habit learning; and the relatively recent development of suitable analysis pipelines for interrogating these relatively small, hardto-visualize structures. We also focused on these regions to reduce multiple comparisons that are hard to mitigate with relatively small sample sizes. The subjects were free from current psychiatric comorbidities and psychotropic medications. Several limitations, however, should be considered. The sample size may have limited the statistical power to detect more subtle differences between the groups (ie, group differences with small effect sizes). Larger samples in future studies would also help to rule out false positives. In addition, the lack of correlation between curvature abnormalities and gambling disorder severity could reflect the smaller sample size for this correlational analysis, as compared with the correlational analysis for impulsiveness, which was measurable along a continuum across all participants. We selected subjects who were not taking psychotropic medications and who were free from comorbid psychiatric conditions including nicotine dependence and substance use. It, therefore, remains to be seen whether the findings generalize to gambling disorder more widely, as the condition is often comorbid with other disorders.

In summary, the current study identified morphologic abnormalities of the pallidum and putamen in people with gambling disorder symptoms (at-risk gambling and gambling disorder), as compared with recreational gamblers without any pathologic symptoms. The right pallidum abnormality bore a particularly strong relationship with trait impulsiveness on the EIQ, rather than with gambling disorder severity. We suggest that pallidum morphology and associated impulsivity might predispose persons to a range of problematic repetitive behaviors including gambling and that these differences observed in people with gambling disorder symptoms may reflect abnormalities of longitudinal brain development related to impulsivity.

\section{Disclosures}

This research was supported by a Center of Excellence in Gambling Research grant from the National Center for Responsible Gaming and a Wellcome Trust Clinical Fellowship (110049/Z/15/Z). Samuel Chamberlain consults for Cambridge Cognition, Shire, and Promentis. There are no competing financial interests in relation to this work. Jon Grant has received research grants from the American Foundation for Suicide Prevention, TLC Foundation, and Takeda Pharmaceuticals. Jon Grant receives yearly compensation from Springer Publishing for acting as Editor-in-Chief of the Journal of Gambling Studies and has received royalties from Oxford University Press; American Psychiatric Publishing, Inc; Norton Press; and McGraw Hill. Masanori Isobe has nothing to disclose.

\section{REFERENCES:}

1. Petry NM, Stinson FS, Grant BF. Comorbidity of DSM-IV pathological gambling and other psychiatric disorders: results from the National Epidemiologic Survey on Alcohol and Related Conditions. J Clin Psychiatry. 2005; 66(5): 564-574.

2. Limbrick-Oldfield EH, van Holst RJ, Clark L. Fronto-striata dysregulation in drug addiction and pathological gambling: consistent inconsistencies? Neuroimage Clin. 2013; 2: 385-393. 
3. Goudriaan AE, Yücel M, van Holst RJ. Getting a grip on problem gambling: what can neuroscience tell us? Front Behav Neurosci. 2014; 8: 141

4. Roca M, Torralva T, López P, Cetkovich M, Clark L, Manes F. Executive functions in pathologic gamblers selected in an ecologic setting. Cogn Behav Neurol. 2008; 21(1): 1-4.

5. Kertzman S, Lowengrub K, Aizer A, Vainder M, Kotler M, Dannon PN. Go-no-go performance in pathological gamblers. Psychiatry Res. 2008; 161(1): 1-10.

6. Odlaug BL, Chamberlain SR, Kim SW, Schreiber LR, Grant JE. A neurocognitive comparison of cognitive flexibility and response inhibition in gamblers with varying degrees of clinical severity. Psychol Med. 2011; 41(10): 2111-2119.

7. Eagle DM, Wong JC, Allan ME, Mar AC, Theobald DE, Robbins TW. Contrasting roles for dopamine D1 and D2 receptor subtypes in the dorsomedial striatum but not the nucleus accumbens core during behavioral inhibition in the stop-signal task in rats. J Neurosci. 2011; 31(20): 7349-7356.

8. Preuschoff K, Bossaerts P, Quartz SR. Neural differentiation of expected reward and risk in human subcortical structures. Neuron. 2006; 51(3): 381-390.

9. Hauser TU, Eldar E, Dolan RJ. Separate mesocortical and mesolimbic pathways encode effort and reward learning signals. Proc Natl Acad Sci USA. 2017; 114(35): E7395-E7404.

10. Elliott R, Friston KJ, Dolan RJ. Dissociable neural responses in human reward systems. J Neurosci. 2000; 20(16): 6159-6165.

11. Linnet J, Peterson E, Doudet DJ, Gjedde A, Møller A. Dopamine release in ventral striatum of pathological gamblers losing money. Acta Psychiatr Scand. 2010; 122(4): 326-333.

12. Joutsa J, Johansson J, Niemelä S, et al. Mesolimbic dopamine release is linked to symptom severity in pathological gambling. Neuroimage. 2012; 60(4): 1992-1999.

13. van Holst RJ, van den Brink W, Veltman DJ, Goudriaan AE. Why gamblers fail to win: a review of cognitive and neuroimaging findings in pathological gambling. Neurosci Biobehav Rev. 2010; 34(1): 87-107.

14. Brevers D, Bechara A, Hermoye L, et al. Comfort for uncertainty in pathological gamblers: a fMRI study. Behav Brain Res. 2015; 278: 262-270.

15. Voon V, Fernagut PO, Wickens J, et al. Chronic dopaminergic stimulation in Parkinson's disease: from dyskinesias to impulse control disorders. Lancet Neurol. 2009; 8(12): 1140-1149.

16. Petersen K, Van Wouwe N, Stark A, et al. Ventral striatal network connectivity reflects reward learning and behavior in patients with Parkinson's disease. Hum Brain Mapp. 2018; 39(1): 509-521.

17. Koehler S, Hasselmann E, Wüstenberg T, Heinz A, Romanczuk Seiferth N. Higher volume of ventral striatum and right prefrontal cortex in pathological gambling. Brain Struct Funct. 2015; 220(1): 469-477.

18. Mohammadi B, Hammer A, Miedl SF, et al. Intertemporal choice behavior is constrained by brain structure in healthy participants and pathological gamblers. Brain Struct Funct. 2016; 221(6): 3157-3170.

19. Fuentes D, Rzezak P, Pereira FR, et al. Mapping brain volumetric abnormalities in never-treated pathological gamblers. Psychiatry Res. 2015; 232(3): 208-213.

20. Patenaude B, Smith SM, Kennedy DN, Jenkinson M. A Bayesian model of shape and appearance for subcortical brain segmentation. Neuroimage. 2011; 56(3): 907-922.

21. Dale AM, Fischl B, Sereno MI. Cortical surface-based analysis. I. Segmentation and surface reconstruction. Neuroimage. 1999; 9(2): 179-194.
22. Fischl B, Sereno MI, Dale AM. Cortical surface-based analysis. II Inflation, flattening, and a surface-based coordinate system. Neuroimage. 1999; 9(2): 195-207.

23. Grant JE, Steinberg MA, Kim SW, Rounsaville BJ, Potenza MN Preliminary validity and reliability testing of a structured clinical interview for pathological gambling. Psychiatry Res. 2004; 128(1): 79-88.

24. American Psychiatric Association. Diagnostic and statistical manual of mental disorders (5th ed.) (DSM-5). Arlington, VA: American Psychiatric Publishing; 2013.

25. Sheehan DV, Lecrubier Y, Sheehan KH, et al. The Mini-International Neuropsychiatric Interview (M.I.N.I.): the development and validation of a structured diagnostic psychiatric interview for DSM-IV and ICD-10. J Clin Psychiatry. 1998; 59(suppl 20): $22-33$.

26. Eysenck HJ, Eysenck SBG. Manual for the Eysenck Personality Questionnaire. London: Hodder \& Stoughton; 1978.

27. Nelson HE. National Adult Reading Test (NART) Manual. Windsor, $\mathrm{UK} ; 1982$.

28. Isobe M, Redden SA, Keuthen NJ, et al. Striatal abnormalities in trichotillomania: a multi-site MRI analysis. Neuroimage Clin. 2018 ; 17: 893-898.

29. Zhang Y, Brady M, Smith S. Segmentation of brain MR images through a hidden Markov random field model and the expectationmaximization algorithm. IEEE Trans Med Imaging. 2001; 20(1): 45-57.

30. Buckner RL, Head D, Parker J, et al. A unified approach for morphometric and functional data analysis in young, old, and demented adults using automated atlas-based head size normalization: reliability and validation against manual measurement of total intracranial volume. Neuroimage. 2004; 23(2): 724-738.

31. Hill K, Bolo N, Sarvode Mothi S, et al. Subcortical surface shape in youth at familial high risk for schizophrenia. Psychiatry Res Neuroimaging. 2017; 267: 36-44.

32. Lu Y, Liang H, Han D, et al. The volumetric and shape changes of the putamen and thalamus in first episode, untreated major depressive disorder. Neuroimage Clin. 2016; 11: 658-666.

33. Winkler AM, Ridgway GR, Webster MA, Smith SM, Nichols TE. Permutation inference for the general linear model. Neuroimage. 2014; 92: 381-397.

34. Black DW, Shaw M, McCormick B, Allen J. Pathological gambling: relationship to obesity, self-reported chronic medical conditions, poor lifestyle choices, and impaired quality of life. Compr Psychiatry. 2013; 54(2): 97-104.

35. Grant JE, Kim SW. Demographic and clinical features of 131 adult pathological gamblers. J Clin Psychiatry. 2001; 62(12): 957-962.

36. Mallet N, Schmidt R, Leventhal D, et al. Arkypallidal Cells Send a Stop Signal to Striatum. Neuron. 2016; 89(2): 308-316.

37. Strassmann G, Sturner W, Helpern M. [Brain lesions, especially lenticular nucleus softening in heroin addicts, barbiturate poisoning, late death after hanging and heart arrest during anesthesia]. Beitr Gerichtl Med. 1969; 25: 236-242.

38. Andersen SN, Skullerud K. Hypoxic/ischaemic brain damage, especially pallidal lesions, in heroin addicts. Forensic Sci Int. 1999; 102(1): 51-59.

39. Tolomeo S, Gray S, Matthews K, Steele JD, Baldacchino A. Multifaceted impairments in impulsivity and brain structural abnormalities in opioid dependence and abstinence. Psychol Med. 2016; 46(13): 2841-2853. 